\title{
Paying Attention to Values and Culture: An Artifact to Support the Evaluation of Interactive Systems
}

\author{
Roberto Pereira \\ University of Campinas (UNICAMP)
}

\author{
Leonardo Cunha de Miranda \\ Federal University of Rio Grande do Norte
}

\begin{abstract}
Evaluating interactive systems is becoming an increasingly complex challenge. As information and communication technology have left the context of offices and workplaces to pervade every aspect of people's personal and social lives, issues such as emotional and affective aspects, sociability, culture and human values are now in play and must be explicitly considered. This situation is very different from what designers and researchers have experienced before, and requires new methods, techniques and tools to support them in producing solutions aligned with the new demands of a digital age. In this paper we focus on values and culture in the evaluation of interactive systems, presenting the eValue - an artifact we created to support designers in a culturally informed and value-oriented evaluation. To situate our discussion in a practical context, we present a case study where the artifact was used to evaluate seven prototypes of social applications for the Brazilian Interactive Digital Television.
\end{abstract}

\section{Introduction}

Interactive systems are a growing reality worldwide and people use them through different devices, for different purposes, in quite different contexts, and with unforeseen and far-reaching consequences. In this sense, as far as technology is present in almost every place, activity, object, and people's life, the task of designing it has assumed new dimensions in terms of complexity, and has required a deep understanding regarding its social and ethical responsibility.

In the context of Human-Computer Interaction (HCI), Sellen et al. [1] argue that our relationship with technology has been profoundly modified in the last years. For the authors, we are increasingly dependent on technology, in the sense that we rely on computers for practically everything in our lives. We are even more hyperconnected and pervaded by communication technologies and devices that put and keep us online everywhere, every time. Our footprints are not ephemeral anymore, in the sense that the lifetime of the information about our lives

\author{
Samuel B. Buchdid \\ University of Campinas (UNICAMP)
}

and actions has been permanently extended. In this new and complex landscape, even the terms "human", "computer" and "interaction" have to be rethought, requiring a deeper understanding of what means to be human, what/where is the computer/interface and how is the interaction in our digital age. This means that, when designing people's interactions with computers and with others by using them, we have to consider users in the widest extension possible, the new forms of interactions that go beyond the task-oriented approach, and the context of use that transcend personal desktop computers.

Winograd [2] highlights that designing interactive systems goes beyond the construction (and evaluation) of an interface to encompass all the interspace in which people live. The author draws attention to the complex interplay among technology, individual psychology and social communication, requiring a shift from seeing the machinery to seeing the lives of the people using it. This shift, in turn, demands attention to relevant factors, such as the emotional and affective ones, and those related to the values and the culture of users, that become hard to quantify and even to identify.

In fact, the factors cited above were traditionally left on the margin of approaches to computer systems development, but now are being moved to the centre, characterizing what Harrison et al. [3] named the third paradigm in the HCI field - and what we prefer to refer to as a new moment in the field. According to the authors, while the first and second HCI paradigms were oriented to issues of ergonomics and cognitive factors, respectively, the third one must deal with the establishment and multiplicity of meaning in situated interactions. In this paradigm, artifacts and their contexts are mutually defining and being subject to different interpretations.

Differently from the two first paradigms in which substantial work and knowledge have been produced since the last three decades, the third one remains quite unexplored (and even not understood) by the HCI academy. Indeed, it requires collaboration with other areas (e.g., anthropology, sociology, education) as well as the development of methods, artifacts, and 
tools for supporting designers in understanding and dealing with its complex nature.

Our research projects have been situated in this scenario, investigating the design of interface and interaction solutions as well as frameworks and resources to support designers in this new $\mathrm{HCI}$ moment.

Values are culturally built [4,5], and dealing with them in the design of technology has been pointed out as a great challenge for the HCI domain [1]. Aiming to shed light on this challenge, we have created/adapted artifacts and methods for supporting designers in what we named a value-oriented and culturally informed approach (VCIA) for design the interested reader may visit [6] for a list of artifacts, their templates, explanations and references. In this paper, we focus on the evaluation of interactive systems and present the eValue: an artifact created to support designers in taking into account the values and the culture of different stakeholders. In order to situate our discussions in a practical setting, we present a case study in which seven prototypes of interactive systems for the Brazilian Interactive Digital Television (iDTV) were evaluated through the eValue. The artifact is grounded on the building blocks of culture [7] and on the Organizational Semiotics theory [8].

This paper is organized as follows: Section 2 introduces the subject of values and culture in technology design. Section 3 presents and explains the eValue artifact. Section 4 presents a case study where the artifact was used to support the evaluation of prototypes. Section 5 discusses the case study's main findings and Section 6 presents our final remarks.

\section{Literature review}

Every technology triggers changes and causes impacts in the environment it is introduced [7]. There are people in that environment who suffer the impact, trigger others, and confer values upon such an innovation [9]. Values are defined by Schwartz [5] as trans-situational goals that vary in importance and serve as principles that guide people's lives and, more generally, by Friedman [10] as something that a person, or a group of people, considers important in life.

Values as well as behavioral patterns, beliefs and preferences are learned and determined by culture $[7,11]$. They are determinants of attitudes, choices and actions, and influence peoples' judgment about products, events and other people. In the context of technology design, Friedman [12] and Bannon [13] suggest that designers communicate values in the solutions they design. However, for Friedman [12] it is not a two-way communication because, unlike people with whom users can disagree and negotiate about values and their meanings, they hardly can do the same with technology. For the author, when designing computer technology it is necessary to see human values from an ethical point of view.

If we consider just a few examples of famous web systems (e.g., Facebook ${ }^{\circledR}, Y_{\text {ouTube }}{ }^{\circledR}$ ), concerns regarding human values and other cultural aspects (e.g., beliefs, behavioral patterns, formal systems) are hardly visible in their design. However, social implications related to values (or to the lack of them) in systems design are being widely reported and can be easily perceived. Winter [14] draws attention to the value of privacy in web applications. Using Facebook $^{\circledR}$ as an example, the author states that privacy issues go from what the application does with users' data to what it allows other applications to do. In another interesting example, Mui [15] reports how pedophiles were using Wikipedia ${ }^{\circledR}$ as a medium to both disseminate their ideas and enter in schools, easily reaching students. Indeed, in practical terms, concern and analysis related to culture and values in technology seem to occur after it has been designed and launched. Consequently, as Knobel and Bowker [16] suggest, most users are faced with undecipherable and unusual decisions already made on their behalf, and often not to their benefit.

In the context cited above, some authors have pointed out the inability of traditional inspection methods, such as the Heuristic Evaluation, to consider and deal with subjective and contextualized issues (e.g., emotional and affective aspects, culture, values) $[17,18]$ as well as characteristics of Web 2.0 applications (e.g., collaboration, user-produced content) [19].In reality, understanding values and culture in order to deal with them is only possible if designers could see the system being designed through the lenses of the different stakeholders and their cultural particularities. However, curricula in Computer Science and Information Technology do not traditionally address methods that enable students to deal with social issues in systems development. In this sense, while the need for considering values and culture in the design process is becoming more and more evident, the inability of current approaches and methods in supporting it is becoming more and more visible.

Some authors have explicitly addressed issues on values in technology design. For instance, Cockton [20] proposed a framework to support what he named a Value-Centred Design (VCD), suggesting some activities and artifacts to support designers in the development of value-centred systems. The focus of VCD is on the understanding of technology design as a process of delivering values. Moreover, since 1996 Friedman [12] has been working on an approach she named Value-Sensitive Design (VSD), intended to support concerns regarding values, especially the ethical ones, in the design of software systems. Other authors have also dealt with the subject of culture in technology design, specially 
investigating cultural factors in usability evaluation and the study of current HCI design methods from a cultural perspective [21, 22, 23].

Nevertheless, Sellen et al. [1] highlight that, despite these efforts, there is still a need for developing and publishing studies to support designers to account for values and deal with the complexity and the different requirements that current technologies have. Indeed, although researches related to Globalization and Internationalization [24] have the premises of not making assumptions based on a single place, culture and values are not often considered. Even the works cited above that look for explicitly dealing with culture and values treat them in isolation. However, as Hall [4] argues, the natural act of thinking is strongly modified by culture. Culture influences what people pay attention to and what they ignore, what people value and what they do not, the way people behave and the way they interpret other's behavior. Therefore, if we are to approach values in systems design, and if we are to support designers in doing so, we must develop solutions (techniques, artifacts, methods, theories) that pay attention to the cultural nature of values, their diversity and complexity.

In the next section we present the eValue: an artifact created to support designers to keep values and culture in mind when analyzing and evaluating interactive systems.

\section{The eValue artifact}

Depending on the way a system is designed, it will afford behaviors that are intrinsically related to individuals and the complex context in which they are using it [25]. As Kolkman [9] argues, if an innovation is inserted in each group of stakeholders accordingly, no serious problems might occur; but sometimes there may be conflicts, and designers will be able to anticipate the reactions of stakeholders only if they could see the world through the lenses of these stakeholders.

However, as designers usually do not have background in social sciences, they might have difficulties in knowing what to do or how to proceed in order to understand and deal with issues related to stakeholders' culture, behaviors, values, conflicts and so on. Consequently, they need concrete artifacts that could inform them during design activities while facilitating their understanding of social issues.

The eValue is an artifact created on the grounds of Organizational Semiotics (OS) theory [8] and the Hall's building blocks of culture [7] — see Figure 1. It is intended to support a culturally informed and value-oriented evaluation of existing applications, or their prototypes.

\begin{tabular}{|c|c|c|c|}
\hline AREA (PMS) & VALUE & I APPLICATION & NOTES \\
\hline \multirow{2}{*}{ 1. Interaction } & 1.1 Identity & & \\
\hline & 1.2 Norms & & \\
\hline \multirow{4}{*}{ 2. Association } & 2.1 Conversation & & \\
\hline & 2.2 Groups & & \\
\hline & 2.3 Relationship & & \\
\hline & 2.4 Trust & & \\
\hline 3. Learning & 3.1 Meta-communication & & \\
\hline \multirow{2}{*}{ 4. Play } & 4.1 Aesthetics & & \\
\hline & 4.2 Emotion and Affection & & \\
\hline \multirow{3}{*}{ 5. Protection } & 5.1 Informed consent & & \\
\hline & 5.2 Reputation & & \\
\hline & 5.3 Security & & \\
\hline \multirow{4}{*}{ 6. Exploitation } & 6.1 Accessibility & & \\
\hline & 6.2 Object & & \\
\hline & 6.3 Property (ownership) & & \\
\hline & 6.4 Usability & & \\
\hline \multirow{3}{*}{ 7. Temporality } & 7.1 Availability & & \\
\hline & 7.2 Awareness & & \\
\hline & 7.3 Presence & & \\
\hline \multirow{4}{*}{ 8. Territoriality } & 8.1 Portability & & \\
\hline & 8.2 Privacy & & \\
\hline & 8.3 Scalability & & \\
\hline & 8.4 Visibility & & \\
\hline \multirow{4}{*}{ 9. Subsistence } & 9.1 Autonomy & & \\
\hline & 9.2 Collaboration & & \\
\hline & 9.3 Reciprocity & & \\
\hline & 9.4 Sharing & & \\
\hline 10. Classification & 10.1 Adaptability & 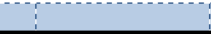 & \\
\hline
\end{tabular}

Figure 1. The structure of the eValue artifact.

The artifact's basic principles are: every interactive system allows users to interact with it and through it, for different purposes and by means of different interface and interaction resources. Depending on the behavior favored or inhibited by the system, it will impact either positively or negatively on users' values related to cultural aspects that pervade everyday life: from the way users learn and play to the way they manage time and space; from the way users interact and associate to the way they work and subsist in the world. The analyst's work consists of exploring the system, questioning and analyzing the way it communicates values and affects users' cultural aspects, taking notes and proposing design alternatives.

In the eValue, the "AREA (PMS)" column presents Hall's [7] ten areas of culture - see Table 1 for an explanation for each area. According to the author, any culture may be mapped through a combination of these areas and develop values with regard to them. For instance, values in association are related to the way society is structured, the groups people form, the role and importance of family, etc. The "VALUE" column presents a list of values we identified in the context of applications for promoting social interaction and classified according to their suitable area [26]. This list is not intended to be definitive or exhaustive. Rather, it is intended to serve as a departure point for analysis as, without previous exposition to cultural issues, it would be harder to proceed if no values were suggested. The column "I" serves to indicate whether the value in its corresponding line was identified in the application being analyzed. The "APPLICATION" column is intended to describe the way the application is reflecting the value. For instance, in the value of 
"Accessibility", evaluators should verify whether the application being analyzed is supporting the value, what features are supporting it, whether they are enough to make the application accessible, etc. Finally, in the "NOTES" column evaluators may register important information regarding the value, highlighting both positive and negative points, benefits and drawbacks, warnings and ideas.

Once filled, the eValue provides a map of what values are being reflected by/on/through the application analyzed and the way it is done. It also presents evaluators' reasoning about each value, pointing out pending questions, critical issues, ideas and possible improvements that may guide a redesign activity, or at least, serve as a reflection list.

Table 1. The building blocks of culture [7].

\section{PMS DESCRIPTION}

Everything people do involves interaction with something/someone else: people, systems, objects, animals, etc. To be alive means to interact with the environment. All the other following areas have 巳 interaction in their nature. As Hall asserts, interaction is at the centre of the universe of culture and everything grows from it.

All living things organize their life in some pattern . of association. This area refers to the different ways that society and its components are organized and \& structured. Governmental and social structures may

$\ll$ vary strongly according to the culture, not only in nature, form and function, but also in importance.

Learning is one of the basic activities present since $\stackrel{00}{\Xi}$ the beginning of life. It has an important role in the E course of man evolution. Education and educational $\stackrel{\circlearrowleft}{\circlearrowleft}$ systems are strongly tied to emotion and as characteristic of a culture as its language.

Funny, emotion and pleasure are terms related to this area. Although its role in the evolution of species is not well understood yet, "Play" is clearly

$\frac{\vec{\alpha}}{2}$ linked to the other areas: in learning it is considered a catalyst; in relationships a desirable characteristic, etc. If one controls the humor of a people, s/he is able to control almost everything else.

Defense is a specialized activity of vital importance. People must defend themselves not only against ฮ hostile forces in nature, but also against those within . human society and internal forces. Cultures have * different mechanisms and strategies of protection

(e.g., medicine, military strategy, religion). Originally named "Defense", we adopted the modification proposed in the OS theory [8].
It is related to the use of materials in order to .0 explore the world. Materials in an environment are st. strongly related to the other aspects of a culture. 을 There are tools and artifacts for cooking, protecting,

I playing, learning, etc. It is impossible to think about a culture with no language and no materials.

Time is related to life in several ways: from cycles, $\stackrel{\nexists}{\nexists}$ periods and rhythms (e.g., breath rate, heartbeat) to measures (e.g., hours, days) and other aspects in 를 society (e.g., division according to age groups, mealtime). The ways people deal with time and the roles of time in society vary across cultures.

It refers to the possession, use and defense of space. Having a territory is essential to life; the lack of a territory is one of the most precarious conditions of life. There are physical (e.g., country, house) as well as social (e.g., social position, hierarchy) and personal spaces (e.g., personal data, office desk). The way space is understood, used and values may vary strongly according to the culture.

It is related to the differences in terms of form and function related to gender. Cultures have different . . forms of distinction and classification and give dif different importance to each one. Originally called "bisexuality", we adopted the name suggested by of the OS theory [8] in order to encompass, beyond differences in gender, the ones related to socioeconomic conditions, age, abilities, etc.

This area includes from people's food habits to the \& economy of a country. Professions, supply chains, deals, natural resources, are all aspects developed in this area and that vary strongly according to the

$\vec{F}$ culture, being influenced not only by the other areas but also by geographical and climatic conditions.

In the next section we describe the case study where the artifact was used to support the evaluation of interactive prototypes focusing on values and culture.

\section{The case study}

This case study was conducted in a Computer Science undergraduate discipline for "Construction of Human-Computer Interfaces" where participants were assigned the task to design social applications for the iDTV. The course took place from August to December, 2011, and by its end, participants had to present a functional prototype of their projects and socialize the final results with the others.

Brazil has more than 190 million inhabitants, a very heterogeneous population in terms of ethnicity, social and economical conditions, and the analogical television is present in $97 \%$ of Brazilian homes [27]. The iDTV is an emergent technology instituted by the Brazilian government in 2003 intending to 
promote social inclusion, cultural diversity, and the native language (Portuguese), for favoring the democratization of information and reduction of economic, geographical and social barriers [28]. In this sense, as Furtado [29] argues, an iDTV application "for all" should consider a comprehensive and contextualized analysis, bringing characteristics of the target audience to the design and evaluation. In fact, designing technologies in such a complex scenario demands a new perspective to the design. It requires what Baranauskas has named [30] and practiced [31, 32] as Socially Aware Computing: "the theory, artifacts and methods we need to articulate to actually make the design socially responsible, participatory and universal as process and product". The eValue is an artifact intended to contribute in this direction.

The case study was conducted with 38 participants divided into 8 groups (from G1 to G8). G1 was formed by the prospective designers: (D1, D2, D3 and D4), G2 (D5..D9), G3 (D10..D14), G4 (D15..D19), G5 (D20..D23), G6 (D24..D28), G7 (D29..D33) and G8 (D34..D38). Finished the course, the groups were asked to voluntarily answer an online questionnaire in order to evaluate the entire process used to design the applications; the participants were also asked permission for using the material they had produced, including their answers to the questionnaire. G1 opted for not answering the questionnaire and is not being included in this analysis.

From the 7 projects: G2 and G6 designed applications for promoting sustainable behavior in its users: a game and a social application, respectively. G3 projected an application to support social interaction over football matches. G4 and G5 designed social network applications for the iDTV. G7 designed an application to support online chat, and G8 designed a platform for interactive online courses through the iDTV.

Before the evaluation activity, each group had produced the first version of an interactive prototype - see Figure 2 for examples. The prototype should allow evaluators to perform at least one complete task relevant to the context of the application. During all the design stage, the concern with the values and culture of stakeholders should be made explicit by the groups. For supporting that, groups: i) were instrumented with artifacts and methods from the OS [8], such as the Stakeholder Identification Diagram, the Value Identification Frame and the Culturally Aware Requirements Framework [33]; ii) applied techniques of design inspired by Participatory Design [34], such as the Brain Drawing; and iii) used Design Patterns specific for iDTV applications [35].

Because the evaluation activity was to be performed, preferentially, by participants from other groups, each group shared: i) all the documentation produced in the previous activities; ii) a brief explanation of the application being designed (the target audience, goals, etc.); and iii) a description of the task to be carried out in the evaluation activity, including its initial and final states. Each group was randomly assigned to evaluate a different project (G1 evaluated the project of G8; G2-G5; G3-G1; G4G4; G5-G2; G6-G7; G7-G6; and G8-G3). Groups received the following instructions for conducting the evaluation activity:

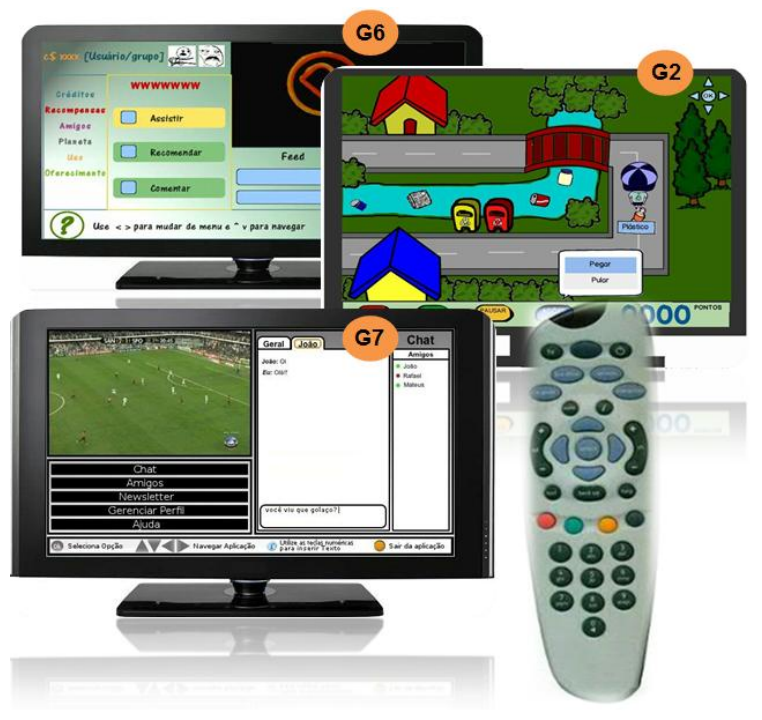

Figure 2. Prototypes designed by G6, G2 and G7.

1. 'Read the description of the application, explore it, and execute the task indicated by its designers'. This step assures evaluators are relatively familiarized with the application, its purpose and features, before starting evaluating it.

2. 'For each value suggested in the eValue artifact, analyze whether there is any feature or attribute of the application that is manifesting/ reflecting/representing the value. In positive situations, indicate it in the corresponding cell ("APPLICATION" column) and mark a "C" in the "I" column indicating that the value was identified in the application, .i.e., it was considered by designers'. This step induces evaluators to think of different values that may be involved in the application and to register that.

3. 'If the value was perceived/identified, discuss whether the application was properly designed to support the value according to the application's purpose, and leave comments, suggestions, and highlights in the "NOTES" column'. Here, evaluators are forced to critically analyze the application according to the values involved, the way they are being involved through design decisions, and the adequateness of such decisions to the application context.

4. 'If the value was not identified, verify whether it is not important to the application or it is being 
neglected/forgotten in the project. In the later case: i) mark an " $\mathrm{N}$ " in the "I" column indicating the value is being neglected; ii) leave your notes to the group drawing attention to its possible impacts; and iii) suggest means of supporting the value in the project'. This step favors evaluators to pay attention to aspects that, although critical, may have been neglected during the design stage. As values have an interactive nature [5], neglecting key values may ignore other similar values as well as promote conflicting and sometimes undesired ones.

As background material for supporting the evaluation activity, each group was supplied with: i) guidelines explaining the activity's steps; ii) the eValue artifact in digital format; iii) a table containing the list of 28 values in the context of social applications [26], a description and an example for each value - see Table 2 for some examples; iv) a simplified explanation of each area of culture (as in Table 1); and v) at least 3 questions related to each area the groups should think about see Table 3.

Table 2.Values in social applications [26].

\section{Value Description}

"Providing access; capable of being reached; also being within reach; easy to communicate or deal with"

Capability of supporting, satisfactorily, a heterogeneous set of users with distinct skills, preferences, needs, perceptual and learning disabilities, and motor and cognitive limitations. Example: the system is fully readable by a screenreader application.

"The distinguishing character or personality of an individual"

The "self" of individuals; the expression of

elements of a person's personality and

individuality (who the person is over space and time). Example: the representation of a person's profile, their activities, personal information, etc

"Consent to surgery by a patient or to participation in a medical experiment by a subject after achieving an understanding of what is involved"

Users' awareness about the possible impacts of their actions. Refers to informing and garnering of people's agreement about what is produced from their interaction with the system and with other

$\Xi$ users. Example: a user agrees to make his/her profile public even after the system alerting about the risks of such a decision.

"The quality or state of being portable". Portable: "usable on many computers with little or no

modification"
$\stackrel{\mathbb{Z}}{2}$ Possibility of using the system, its features and

functionalities, through different devices and on different platforms. Ex: access through mobile phone, compatibility with different browsers.

(...) (...)
Table 3. Questions embedded into the eValue.

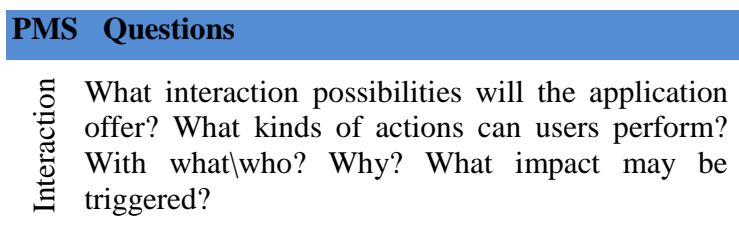

Is the application usage individual or collective? Is .. there any dependence on other organizations/ entities (e.g., data supply)? May it cause impact on any aspect of collective life?

of Is it required any prior knowledge for learning how

$\Xi$ to use the application? What? What is the cognitive effort to learn? What kind of learning it may provide to users?

What kind of emotions the application may/should evoke/avoid (e.g., fun, challenge, warning)? Why?

$\underset{\pi}{*}$ How the application was designed to promote/inhibit these emotions? What are the possible impacts on users?

๘ Can the application compromise users' safety?

What are its policy and terms of use? What are the

features that favor interaction, application, and data security?

. What are the physical devices required to interact with/through the application? Is it required any other material or modification in the environment (e.g., sound, media, lighting, accessory)?

What is the expected frequency of use (daily, weekly, monthly)? If daily, are there strategies for

. promoting users' participation? What about the interaction duration? Is it brief (specific and low

time-consuming tasks), medium (interactions that

$\stackrel{\Xi}{\ominus}$ require a considerable effort) or long (interactions that require a deeper involvement, attention and time)? What are the possible consequences?

In which space will the application be used? What kind of impact may be generated? Is it local/distributed? Can it be collaboratively used? What are the features/resources supporting that?

What is the application's actual utility and applicability? Are the technologies necessary to develop the application open source? Is its final cost (including the physical devices) viable/accessible for the different users' socioeconomic conditions?

Is the application suitable for its target audience? एँ Is it required any adaptation to attend to users' diversity? What are being offered? Are they क enough?

At the end of the evaluation activity, each group received a list of problems and notes, as well as suggestions and ideas, related to values and cultural aspects they should consider in their projects - 
Figure 3 shows an example. After the evaluation activity, groups were instructed to discuss the feedback they received, and to redesign their prototypes taking into account the points they found relevant and arguing about the ones they did not consider.

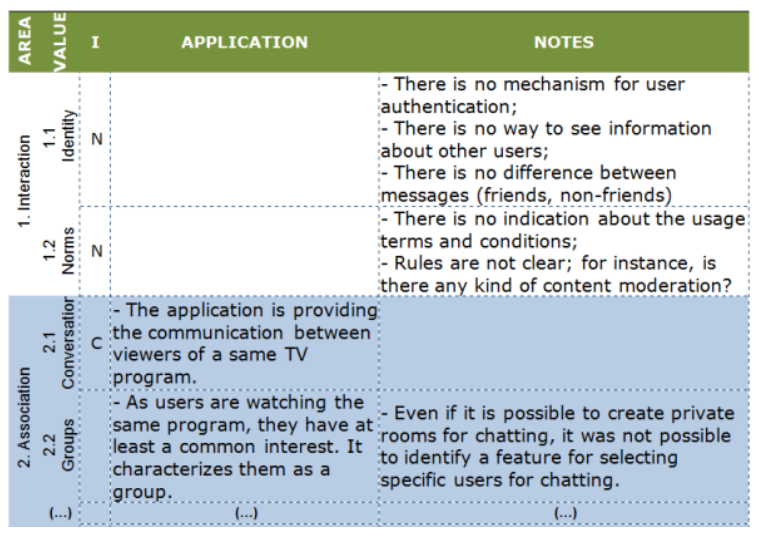

Figure 3. The artifact filled by G6 for G7 - translated by the authors.

\section{Discussion}

During the design stage, groups used other artifacts specially created/adapted to support: i) the identification of the different stakeholders; ii) the values each stakeholder would bring to the project; and iii) the functional and non-functional requirements to support these values - the interested reader may consult [33] for more details. The main goals of the evaluation activity were to inspect whether the prototypes were designed in conformity to the requirements related to values and cultural aspects, and to provide feedback for redesigning the prototypes in order to support these values and cultural aspects accordingly.

Figure 4 presents a synthesis of the results from the evaluation activity. Each group is represented by a column in the "GROUPS" section, and each value corresponds to a line. The letter " $\mathrm{C}$ " in a cell indicates that the corresponding value is being considered by the corresponding group in its project, while the letter " $N$ " indicates that the value is being neglected. Cells in blank indicate the value was not considered relevant to the project by both its designers and evaluators. The last line in the table indicates the groups that evaluated each project.

The " $C$ " indicating that the value was considered does not mean it is being satisfactorily supported, but that the concern with it was made explicit in the project. Evaluators were encouraged to leave their impressions, suggestions and comments regarding the way the value was being handled. For instance, see the value "2.2 Groups" in Figure 3. Evaluators identified it being manifested in the prototype through a feature where users watching the same program would be able to talk to each other by means of synchronous messages. For them, it represents a group of people that has at least a common interest: the program they are watching. However, they pointed out the limitation of the feature regarding private conversations, and we extend it to the problem of information overload. In fact, if a high number of people begin to use the application, it will be harder to interact with specific users, and it will be impossible to control the visibility/privacy of the content being shared. In turn, it also may be a problem of system scalability as well as of users' security and privacy.

\begin{tabular}{|c|c|c|c|c|c|c|c|}
\hline \multirow{2}{*}{ AREA (PMS) } & \multirow{2}{*}{ VALUE } & \multicolumn{6}{|c|}{ GROUPS } \\
\hline & & G2 & G3 & G4 & G5 & G6 & G7 \\
\hline \multirow{2}{*}{ 1. Interaction } & 1.1 Identity & C & C & C & C & C & $\mathbf{N}$ \\
\hline & 1.2 Norms & & C & $\mathbf{N}$ & $\mathbf{N}$ & $\mathbf{N}$ & $\mathbf{N}$ \\
\hline \multirow{4}{*}{ 2. Association } & 2.1 Conversation & & C & C & C & C & C \\
\hline & 2.2 Groups & & & $\mathbf{N}$ & & C & C \\
\hline & 2.3 Relationship & & C & C & C & C & C \\
\hline & 2.4 Trust & C & C & C & & C & C \\
\hline 3. Learning & 3.1 Meta-communication & C & C & C & C & $\mathbf{N}$ & $\mathbf{N}$ \\
\hline \multirow{2}{*}{ 4. Play } & 4.1 Aesthetics & C & C & C & $\mathrm{C}$ & & C \\
\hline & 4.2 Emotion and Affection & c & C & C & c & C & C \\
\hline \multirow{3}{*}{ 5. Protection } & 5.1 Informed consent & $\mathbf{N}$ & $\mathbf{N}$ & $\mathbf{N}$ & $\mathbf{N}$ & C & $\mathbf{N}$ \\
\hline & 5.2 Reputation & & C & C & & $\mathbf{N}$ & $\mathbf{N}$ \\
\hline & 5.3 Security & $\mathbf{N}$ & C & $\mathrm{C}$ & & C & $\mathbf{N}$ \\
\hline \multirow{4}{*}{ 6. Exploitation } & 6.1 Accessibility & C & $\mathbf{N}$ & $\mathbf{N}$ & & C & $\mathbf{N}$ \\
\hline & 6.2 Object & C & C & C & & $\mathbf{N}$ & \\
\hline & 6.3 Property (ownership) & C & C & $\mathbf{N}$ & $\mathrm{C}$ & $\mathbf{N}$ & $\mathbf{N}$ \\
\hline & 6.4 Usability & C & C & C & C & C & C \\
\hline \multirow{3}{*}{ 7. Temporality } & 7.1 Availability & C & & C & & C & \\
\hline & 7.2 Awareness & $\mathbf{N}$ & C & C & & C & $\mathbf{N}$ \\
\hline & 7.3 Presence & & $\mathbf{N}$ & $\mathbf{N}$ & C & $\mathbf{N}$ & $\mathbf{N}$ \\
\hline \multirow{4}{*}{ 8. Territoriality } & 8.1 Portability & & $\mathbf{N}$ & $\mathbf{N}$ & & $\mathbf{N}$ & \\
\hline & 8.2 Privacy & & C & C & $\mathbf{N}$ & C & $\mathbf{N}$ \\
\hline & 8.3 Scalability & & $\mathbf{N}$ & $\mathbf{N}$ & & C & \\
\hline & 8.4 Visibility & $\mathbf{N}$ & & $\mathbf{N}$ & & $\mathbf{N}$ & $\mathbf{N}$ \\
\hline \multirow{4}{*}{ 9. Subsistence } & 9.1 Autonomy & & $\mathbf{N}$ & $\mathbf{N}$ & & C & \\
\hline & 9.2 Collaboration & & & C & & $\mathbf{N}$ & $\mathbf{N}$ \\
\hline & 9.3 Reciprocity & C & C & $\mathbf{N}$ & & C & \\
\hline & 9.4 Sharing & $\mathbf{N}$ & & C & C & C & C \\
\hline \multirow[t]{2}{*}{ 10. Classification } & 10.1 Adaptability & $\mathbf{N}$ & $\mathbf{N}$ & $\mathbf{N}$ & C & $\mathbf{N}$ & $\mathbf{N}$ \\
\hline & Evaluated by: & G5 & G8 & G4 & G2 & G7 & G6 \\
\hline
\end{tabular}

Figure 4. Sumary of the evaluation results.

The "N" indicating that the corresponding value was neglected means evaluators found it critical to consider and deal with it in an explicit way whatever their reasons. It may also mean that they identified a gap between the project's documentation and the final prototype. For instance, see the value "1.2 Norms" in Figure 3. Evaluators start questioning about the rules for using the application because there was no indication that designers thought about them. They also drew attention to the need for clear rules and protocols for interaction, because depending on the target audience, the period in which the application will be used, and the amount of information being shared, it may be necessary a feature to guarantee the quality, integrity and security of interactions (e.g., filtering messages with offensive content).

As we cited previously, some authors have argued that traditional usability inspection methods do not allow evaluators to identify typical problems of social systems, such as the ones related to the content 
produced by users and to the protocols of social interaction [18,19]. Figure 4 shows that five of six groups of evaluators drew attention to the value of "1.2 Norms", and that four concluded it was being neglected by designers. Considering that G6 and G7 evaluated the project of each other, and that G4 evaluated its own project, the fact of the three groups were neglecting the value of "Norms" in their prototypes, but identified it being neglected when acting as evaluators, indicates the ability of the eValue artifact in favoring the critical thinking and the analysis of design decisions that may impact on values.

Figure 4 also allows the identification of interesting points regarding the values involved in the projects. For instance, "Emotion and Affection" and "Usability" were identified by evaluators in all the six projects. It suggests that the concern with such values was present during the design stage, although it does not guarantee they were satisfactorily considered. The values of "Identity", "Relationship", "Conversation", and "Trust", were also identified in the majority of the projects. This was expected because the projects were all intended to promote social interaction among iDTV users.

On the other hand, the value of "Accessibility" was identified in only two projects, indicating that the other four groups did not pay attention to it. It reinforces our observation in [36] that the concept of accessibility is not often considered in the literature as well as in practical contexts. Additionally, the values of "Informed Consent" and "Adaptability" were being considered by one group only, and neglected by the others. Friedman et al. [10] indicate that systems neglect the value of "Informed Consent" because they usually do not let users aware of the consequences triggered by their actions. Neris et al. [37], in turn, suggest that the value of "Adaptability" should be considered in order to develop applications capable of supporting a heterogeneous group of users in their diversity of abilities, preferences, cognitive and motor limitations, etc. In this sense, these values seem to be critical when designing applications for a complex context such as the one in this case study, and Figure 4 shows that, although designers did not pay attention to them when designing their prototypes, they were forced to rethink and review their decisions when acting as evaluators.

According to our description of the case study, designers were instructed to keep values in mind when they were prototyping their solutions, and used techniques and artifacts to support it. Nevertheless, Figure 4 shows that all the groups were neglecting at least one value. Moreover, values and culture must be explicitly considered during all the design stages, from the problem clarification to the system development, deployment and usage.

Finally, although further exposition of the artifact to other people and professional designers in different contexts is still needed, the results and observations from this case study indicate eValue as a promising artifact for supporting a value-oriented and culturally aware evaluation of interactive systems.

\section{Conclusion}

This paper discussed the evaluation of interactive systems in the context of a new moment in the HCI field: a moment where values, emotion, motivation, and several other cultural aspects play a central role, and where new theories, approaches, artifacts and tools for supporting designers when evaluating systems are needed. In this scenario, we presented the eValue, an artifact created on the grounds of Organizational Semiotics and the building blocks of culture, as a way of supporting designers in evaluation activities.

For situating our discussions in a practical context, we presented a case study where the eValue artifact was used to support the evaluation of interactive prototypes of social applications for the iDTV. The case study indicated that the artifact favors attention to issues such as social norms, emotion and affection that, according to the literature, are not emphasized by traditional inspection methods. The results do not suggest the artifact should substitute well-established evaluation techniques, but rather, indicate it may be used as a complement to cover a value-oriented approach in the evaluation of interactive systems.

Finally, this work is intended to contribute with practical solutions that may guide researchers and practitioners in academic as well as industrial settings to account for values and cultural aspects in technology design.

\section{Acknowledgements}

This research is partially funded by $\mathrm{CNPq}$ through the EcoWeb Project (\#560044/2010-0) and by FAPESP (\#2009/11888-7). The authors specially thank the participants of the case study who voluntarily collaborated and authorized the use of the documentation of their projects in this paper.

The present paper is a reviewed and extended version of a previous work published at i-Society 2012 - International Conference for Information Society, entitled "Considering Values and Cultural Aspects in the Evaluation of Interactive Systems Prototypes". The authors thank IJI editors for the invitation and anonymous reviewers for their feedback. 


\section{References}

[1] Sellen, A., Rogers, Y., Harper, R. and Rodden, T. "Reflecting human values in the digital age", Communications of the ACM, Vol. 52, pp. 58-66, 2009.

[2] Winograd, T. "The design of interaction". In Beyond Calculation: The Next Fifty Years of Computing, SpringerVerlag, pp.149-161, 1997.

[3] Harrison, S., Tatar D. and Sengers, P. "The three paradigms of HCI”, ACM AltCHI'07, pp.1-21, 2007.

[4] Hall, E.T. "Beyond culture”, Anchor Books, 1977.

[5] Schwartz, S.H. "Basic human values: their content and structure across countries". Values and Behaviors in Organizations. Vozes, 2005.

[6] "Artifacts for VCIA". http://www.nied.unicamp.br/ ecoweb/products/artifacts. on Aug 16, 2012.

[7] Hall, E.T. "The silent language", Anchoor Books, 1959.

[8] Liu, K. "Semiotics in information systems engineering”, Cambridge, University Press, 2000.

[9] Kolkman, M. "Problem articulation methodology", $\mathrm{PhD}$ thesis, University of Twente, Enschede, 1993.

[10] Friedman, B., Kahn, P.H. and Borning, A. "Value sensitive design and information systems". HumanComputer Interaction and Management Information Systems: Foundations, Armonk, pp. 348-372, 2006.

[11] Rokeach, M. "The nature of human values", New York Free Press, 1973.

[12] Friedman, B. "Value-sensitive design", Interactions, pp. 16-23, 1996

[13] Bannon, L. "Reimagining HCI: toward a more human-centered perspective", Interactions, Vol 18(4), pp. $50-57,2011$.

[14] Winter, J. "Pedophiles find a home on wikipedia". Fox News. http://www.foxnews.com/scitech/2010/06/25/ex clusive-pedophiles-find-home-on-wikipedial, on Aug 19, 2012.

[15] Mui, C. "Facebook's privacy issues are even deeper than we knew". Forbes. http://www.forbes.com/sites/chun kamui/2011/08/08/facebooks-privacy-issues-are-evendeeper-than-we-knew/, on Aug 19, 2012.

[16] Knobel, K. and Bowker, G.C. "Values in design", Communications of the ACM, Vol 54(7), pp. 26-28, 2011.

[17] Hart, J., Ridley, C., Taher, F., Sas, C. and Dix, A "Exploring the facebook experience: A New Approach to Usability", In NordiCHI 2008, ACM Press, pp. 471-474, 2008.
[18] Silva, P.A. and Dix, A. "Usability - not as we know it!", In 21st BCS Conference on Human-Computer Interaction, pp. 103-106, 2007.

[19] Thompson, A-J. and Kemp, E.A. "Web 2.0: extending the framework for heuristic evaluation", In ACM CHINZ 2009, Auckland, pp. 29-36, 2009.

[20] Cockton, G. "A development framework for valuecentred design". In ACM CHI 2005, Oregon, USA, pp. 1292-1295, 2005.

[21] Winschiers H. and Fendler, J. "Assumptions considered harmful: The Need to Redefine Usability", In 2nd International Conference on Usability and Internationalization, pp. 22-27, 2007.

[22] Del Gado, E. and Nielsen, J. "International users interface", New York: John Wiley, 1996.

[23] Maunder, A.; Marsden, G.; Gruijters, D. and Blake, E. "Designing Interactive Systems for the Developing World - Reflections on User-Centered Design". In Proceedings of the 2nd Conference on Information Communications Technologies and Development (ICTD ‘07). 2007.

[24] A. Marcus, "International and intercultural user interfaces". In Stephanidis, C. (Ed.), Users Interfaces for All: Concepts, Methods and Tools, Lawrence Erlbaum, pp. 47-63, 2001

[25] Pereira, R., Baranauskas, M.C.C. and Almeida, L.D. "The value of value identification in web applications". In IADIS International Conference on WWW/Internet (ICWI), pp. 37-44, 2011.

[26] Pereira, R. and Baranauskas, M.C.C. "Seeing Social Software Analysis and Evaluation through the Lenses of Culture". In R. Zhang et al. (Eds.): ICEIS 2011, Springer LNBIP 102, pp. 374-387, 2012.

[27] IBGE. "National survey by household sample". http://www.ibge.gov.br /home/download/estatistica.shtm, on Aug 19, 2012.

[28] Brasil, "Presidential decree No 4.901", http://www.planalto.gov.br/ccivil_03/decreto/2003/d4901. htm, on Aug 19, 2012.

[29] Furtado, E., Mayora-Ibarra, O., Fuhrhop, C. "Assessments and Consensus on Users' Needs of CrossCultural Stakeholders: Expectations on 'Being Part of a Digital TV Project!'”, in EuroITV '10 Proceedings of the eigth european conference on European interactive television conference, pp. 197-204, ACM Press, 2010.

[30] Baranauskas, M.C.C. "Socially aware computing", in VI International Conference on Engineering and Computer Education, pp. 1-4, 2009.

[31] Baranauskas, M.C.C. and Bonacin, R. "Design Indicating Through Signs". MIT Press Design Issues, Vol. 24 (1), pp. 30-45. 2008.

[32] Miranda, L.C., Hornung, H.H. and Baranauskas,M.C.C. "Adjustable interactive rings for 
iDTV,". In: IEEE Transactions on Consumer Electronics (IEEE T-CE), Vol. 56 (3), pp. 1988-1996, 2010.

[33] Pereira, R., Buchdid S.B. and Baranauskas, M.C.C. "Keeping Values in Mind: Artifacts for a Value-Oriented and Culturally Informed Design”. In 14th International Conference on Enterprise Information Systems (ICEIS 2012). pp. 25-34. 2012.

[34] Schuler, D. and Namioka, A. "Participatory design: principles and practices", Hillsdale, Lawrence Erlbaum Associates, Inc., 1993.

[35] Kunert, T. "User-centered interaction design patterns for interactive digital television applications", Springer, 2009.

[36] Pereira, R., Baranauskas, M.C.C. and Silva, S.R.P. “A discussion on social software: concept, building blocks and challenges, International Journal for Infonomics (IJI), Vol. 3 (4), pp. 533-542, 2010.

[37] Neris, V.P.A. and Baranauskas, M.C.C. "End-user tailoring: a semiotic-informed perspective", In ICOS' 2007 International Conference on Organizational Semiotics, pp. 47-53, 2007. 